\title{
Precrash Dipping Nose (PCDN) Needs Pedestrian Recognition
}

\author{
Ho Gi Jung, Member, IEEE, Byung Moon Kwak, Jeong Soo Shim, Pal Joo Yoon, and Jaihie Kim
}

\begin{abstract}
This paper investigates the effect of vehicle front height lowering operation of precrash dipping nose (PCDN) on pedestrians. Although PCDN was developed for vehicle-vehicle side crashes, there is a possibility that the range sensor for crash detection will fail to distinguish a group of pedestrians from a side-faced vehicle. For simulation-based investigation, a vehicle model, an air spring with PCDN actuator, a pedestrian, and active hood system (AHS) were modeled. Two vehicle models were made for a sedan and a sport utility vehicle (SUV), respectively. In all crash situations (frontal crash without AHS, frontal crash with AHS, and side crash without AHS), a falsely operated PCDN is expected to cause worse pedestrian injury. As a conclusion, we insist that PCDN should incorporate a pedestrian-recognition capability into its crash detection system to reduce the improper activation of its actuator. In addition, as AHS showed a good performance even in improper activation, we propose a complementary method that activates AHS when PCDN is activated. It is noteworthy that the increasing importance of pedestrian protection seems to enforce even a system developed for vehicle-vehicle crashes to be investigated from the perspective of pedestrian safety.
\end{abstract}

Index Terms-Crash simulation, pedestrian protection, pedestrian recognition, precrash dipping nose (PCDN), side impact countermeasure.

\section{INTRODUCTION}

$\mathbf{S}$ IDE IMPACTS have always been a real challenge for the automotive industry due to their particularities: small survival space, few energy-absorbing structures, and little time and space to ensure the maximum functionality of restraint systems [1]. According to the Insurance Institute for Highway Safety (IIHS) statistics, in the U.S., each year more than 9000 passenger vehicle occupants die as a result of side impacts. Head injuries are the leading cause. The increasing number of high-riding vehicles on the road these days increases this risk, making it more likely that the front end of a striking vehicle in a side impact will make contact with the heads of occupants in the vehicle that is struck [2]. To reduce the risk of serious and

Manuscript received February 2, 2008; revised August 25, 2008 and September 19, 2008. Current version published December 1, 2008. The Associate Editor for this paper was A. Eskandarian.

H. G. Jung is with MANDO Corporation Global R\&D H.Q., Yongin 446901, Korea, and also with the School of Electrical and Electronic Engineering, Yonsei University, Seoul 120-749, Korea (e-mail: hgjung@mando.com; hgjung@yonsei.ac.kr).

B. M. Kwak and J. S. Shim are with TUR Consulting Corporation, Suwon 443-813, Korea (e-mail: bmkwak@turc.co.kr; jsshim@turc.co.kr).

P. J. Yoon is with MANDO Corporation Global R\&D H.Q., Yongin 446-901, Korea (e-mail: pjyoon@mando.com).

J. Kim is with the School of Electrical and Electronic Engineering, Yonsei University, Seoul 120-749, Korea (e-mail: jhkim@yonsei.ac.kr).

Color versions of one or more of the figures in this paper are available online at http://ieeexplore.ieee.org.

Digital Object Identifier 10.1109/TITS.2008.2006789 fatal injuries to passengers in side impact collisions, specific regulations pertaining to this matter such as the Federal Motor Vehicle Safety Standards (FMVSS) 214 and European Union (EU) Directive 96/27/EC have been established in each nation. In addition, national agencies also perform the Side Impact New Car Assessment Program (SINCAP), New Car Assessment Program (NCAP), Offset NCAP (OFFCAP), and other rigorous tests on cars being domestically sold, the results of which are made public to help people decide which automobile is the safest [3].

Countermeasures for side impact can be divided into three approaches, i.e., reinforcement of side structure, restraint system, and vehicle height adjustment. Reinforcement of side structure includes reinforced material [3], impact force distributing structure [4], and reinforcing actuator [1]. The restraint system includes side and roof airbag. IIHS researchers concluded that side airbags protecting the torso reduced deaths by $26 \%$, and side airbags protecting the head reduced deaths by $37 \%$ [2], [5]. Vehicle height adjustment prevents the struck vehicle from being impacted at its weakest side structure, i.e., the door [6]-[9]. Instead, if the system detects an impending crash via range sensors, it adjusts the level of the vehicle about to be struck [6], [7] or the striking vehicle [8], [9] to cause the crash to occur at stronger side structures such as the bumper and door sill. Volkswagen and Daimler Chrysler have developed and installed systems on the struck vehicle using an air spring with a high pressure tank or hydraulic actuator of active body control (ABC), respectively [6], [7]. Reversibly, Autoliv has introduced and installed a system on the striking vehicle, named precrash dipping nose (PCDN) [8], [9]. When the striking vehicle with PCDN detects an impending side collision, it levels down its front end; in other words, it dives by way of a rapid exhaust valve.

Although PCDN is supposed to give the occupants in the passenger car a four times better chance of surviving [8], what effect PCDN will produce on pedestrians has not been investigated. It is this problem that is dealt with in this paper. Considering that a set of 10-15 different features of radar signal were needed to classify an object into three types, i.e., vehicle, pedestrian, and stationary object [10], then the precrash radar of [8] is thought to be insufficient to recognize the type of object. Although Ford developed a sensor fusion-based vehicle detection that is able to classify the vehicle type to more precisely predict collision, it also did not consider the case when the object was a pedestrian [11]. In fact, the risk of false positive was already mentioned in [9]. There exists the possibility for PCDN to falsely recognize a group of pedestrians as a side-faced vehicle, thereby conducting front-end level down operation. 
Although a system successfully operates in targeted situations, it can cause serious results in the case far from the average situations and in unconsidered situations. We already know one good example: the tragedy of airbag fatalities to children and short drivers [12]. Although the introduction of airbags significantly improves the safety of the occupant, airbags can also cause fatal injuries when the occupant is a child or a short driver. According to the National Highway Transportation and Safety Administration (NHTSA), in the U.S., between 1986 and 2001, 19 infants, 85 children, and seven adults were killed by airbags during relatively minor vehicle collisions. The main cause of these tragedies was that airbag developers considered only heavy male adults. After these tragedies, NHTSA defined FMVSS 208, which mandates automatic airbag suppression in May 2001 [12].

Pedestrian safety is an important problem of global dimension. Pedestrians account for $65 \%$ of the fatalities out of the 1.17 million traffic-related deaths across the world, with $35 \%$ of these being children [13]. In this sense, not only pedestrian safety systems like the Advanced Protection For Vulnerable Road Users (APVRU) [14] but, in addition, safety systems unrelated to pedestrians should be investigated from the viewpoint of pedestrian safety. This proposal is the generalized conclusion of this paper. As a start, we investigated the effect of PCDN on pedestrians by simulation and found that the false operation of PCDN gave a detrimental effect to pedestrians. As a result, we conclude that a more intensive investigation is needed and that PCDN should incorporate pedestrian recognition into its sensing system.

\section{Simulation Model}

\section{A. Vehicle Model}

For simulation-based investigation, a full vehicle dynamic model was constructed with ADAM/View version 2005R2 [15]. The vehicle model has 123 kinematical degrees of freedoms (DOF). Suspension systems were modeled by a MacPherson strut for the front and multilink for the rear, respectively. Tires were modeled by MF-tire, whereas the relaxation and braking system was modeled by the semi anti-lock braking system (ABS) that implemented slip ratio control. Bump and rebound stoppers were modeled by AKIMA spline, and its free stroke was set to $106.7 \mathrm{~mm}$, as shown in Fig. 1. Because the simulation results were expected to be different according to vehicle type, two vehicle types, i.e., sedan and sport utility vehicle (SUV), were modeled using the real parameter values of the Hyundai Motor Company (HMC) sedan and SUV.

\section{B. Air Spring Model}

The air spring was modeled by an air chamber with two orifices, as shown in Fig. 2. $P_{2}$ denotes the pressure of the air chamber. $P_{1}$ and $P_{3}$ denote the pressure of a high-pressed air tank and atmosphere, respectively. $A_{12}$ denotes the orifice area between the air tank and the air chamber, and $A_{23}$ denotes the orifice area between the air chamber and the atmosphere. The used specifications of coil spring are as follows: spring

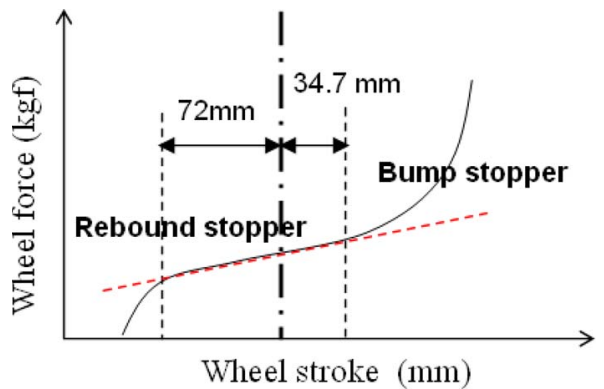

Fig. 1. Relation between wheel stroke and wheel force.

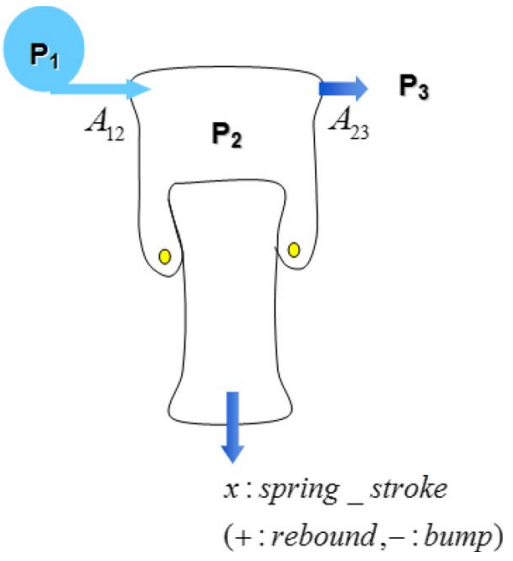

Fig. 2. Air spring model.

constant $k=3.2 \mathrm{kgf} / \mathrm{mm}$, static load $F_{s}=521 \mathrm{kgf}$, and free length $\lambda=401.53 \mathrm{~mm}$. To make the air spring compatible with the coil spring, the parameters of the air spring were established: effective area $A_{\text {eff }}=100 \mathrm{~cm}^{2}$, initial volume $V_{0}=\gamma$. $F_{s} \cdot A_{\text {eff }} / k=2260 \mathrm{~cm}^{3}$, and initial pressure $P_{20}=F_{s} / A_{\text {eff }}=$ 5.21 bar.

The dynamics of the air spring were modeled by the mass fluid equation

$$
\dot{m}_{2}=C_{d} \cdot K \cdot\left(A_{12} \cdot \frac{P_{1}}{\sqrt{T_{1}}} \cdot N_{12}-A_{23} \cdot \frac{P_{2}}{\sqrt{T_{2}}} \cdot N_{23}\right)
$$

where

$$
N_{i j}= \begin{cases}\left(\frac{\left(\frac{P_{j}}{P_{i}}\right)^{\frac{2}{\gamma}}-\left(\frac{P_{j}}{P_{i}}\right)^{\frac{(\gamma+1)}{\gamma}}}{\frac{(\gamma-1)}{2} \cdot\left(\frac{2}{\gamma+1}\right)^{\frac{(\gamma+1)}{(\gamma-1)}}}\right)^{1 / 2}, & \text { if }\left(P_{j} / P_{i}\right) \geq b_{c r} \\ =1.0, & \text { if }\left(P_{j} / P_{i}\right) \leq b_{c r}\end{cases}
$$

and, respectively, by the chamber pressure equation and the chamber volume difference and temperature equation

$$
\begin{aligned}
& \dot{P}_{2}=-\frac{\gamma \cdot P_{2}}{V_{2}} \cdot \dot{V}_{2}+\frac{\gamma \cdot R \cdot T_{2}}{V_{2}} \cdot \dot{m}_{2} \\
& \dot{V}_{2}=A_{\text {eff }} \cdot \dot{x} \quad T_{2}=T_{20} \cdot\left(\frac{P_{2}}{P_{20}}\right)^{\frac{(\gamma-1)}{\gamma}} .
\end{aligned}
$$

The used constants are the coefficient of discharge $C_{d}=$ 0.85 , gas constant $R=285.55, \gamma=1.39$, reservoir (tank) pressure $P_{1}=10.0 \mathrm{bar}$, atmospheric pressure $P_{3}=1.0 \mathrm{bar}$, initial 
temperature $T_{1}=T_{20}=T_{3}=293^{\circ} \mathrm{K}$, initial gas mass $m_{20}=$ $P_{20} \cdot V_{20} / R / T_{20}$, and critical pressure ratio $b_{c r}=(2 /(\gamma+$ $1))^{\gamma /(\gamma-1)}, K=\left((\gamma / R) \cdot(2 /(\gamma+1))^{(\gamma+1) /(\gamma-1)}\right)^{1 / 2}$.

The operation of the air spring for PCDN, known as puncture hereafter, was modeled by rapidly opening the orifice between the air chamber and the atmosphere. It can be expressed as a step function of orifice area $A_{23}$. The rising time is set to $50 \mathrm{~ms}$, and the final value is the maximum value of $A_{23}$. Fig. 3(a) and (b) shows the vertical displacement $x$ of the air spring and the vehicle longitudinal acceleration of the SUV and sedan, respectively. In each figure, the solid lines correspond to the case with PCDN, and the dotted lines correspond to the case without PCDN. In Fig. 3(a), it can be observed that PCDN lowers the vertical displacement of the air spring by $29.3 \mathrm{~mm}$ compared with the normal case. In Fig. 3(b), the maximum displacement of the sedan is measured as $42.6 \mathrm{~mm}$.

\section{AHS Model}

According to the analysis of vehicle impact zones in pedestrian-vehicle collisions, vehicle fronts are responsible for most pedestrian injuries; $71.1 \%$ according to U.S. NHTSA data. Just about $80 \%$ of the fatalities occur when the pedestrian's head hits the hood or windshield, whereas most of the remaining fatalities occur when the pedestrian is hurled to the street [16]. The European Enhanced Vehicle Safety Committee (EEVC) Working Group (WG) 10 and WG 17 has developed test procedures to assess the level of pedestrian protection for vehicle fronts. Based on the EEVC WG 17 report, legal requirements have been derived. The European directive (2003/102/EC) consists of head impact, upper leg impact, and lower leg impact. The requirement will be enforced in two phases, i.e., 2005 and 2010 [17].

Theoretically, around $55 \mathrm{~mm}$ of stopping distance is needed at an impact speed of $40 \mathrm{~km} / \mathrm{h}$ to be able to keep the head injury criterion (HIC) value below 1000 for an adult headform. To meet this requirement, active hood system (AHS), or active bonnet, was introduced by Autoliv. The system comprises two lifting elements that lift the rear corners of the bonnet. The lifting elements consist of compressed metal bellows that are filled with gas from microgas generators in the event of an impact [18]. In our simulations, AHS was modeled by a rotational hinge and lifting actuator, which were installed at the lower part of the windshield, and it extended its free length by $115 \mathrm{~mm}$ within $50 \mathrm{~ms}$ after crash, maintaining the spring force [19], [20]. Fig. 4 shows the structure and operation of the modeled AHS. One AHS model was used for both SUV and sedan. Because the traditional hood inner design with rib structure, which usually has weak points and stiff points, tends to be replaced by novel designs with even stiffness all over the hood area [17], we assumed that the hood structure had a uniform stiffness on its entire surface.

\section{Pedestrian Model}

A pedestrian was modeled using the Biomechanics Research Group, Inc. (BRG) LifeMOD, which is a plugin of the ADAMS and provides an environment to define the task of creating
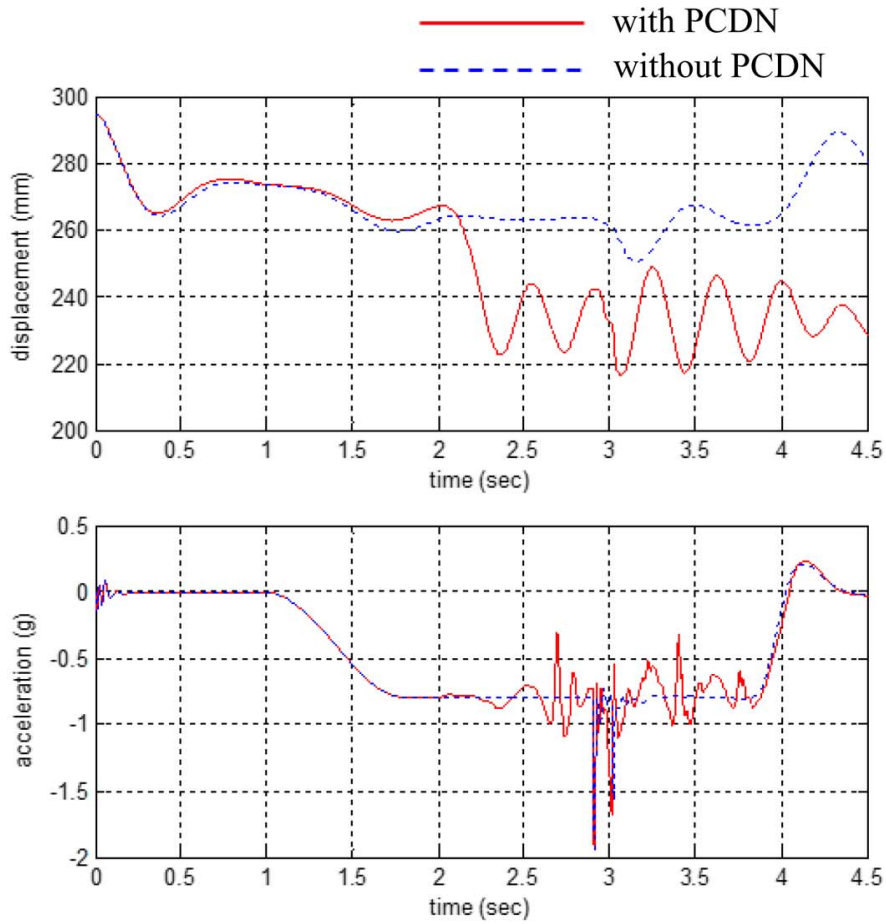

(a)
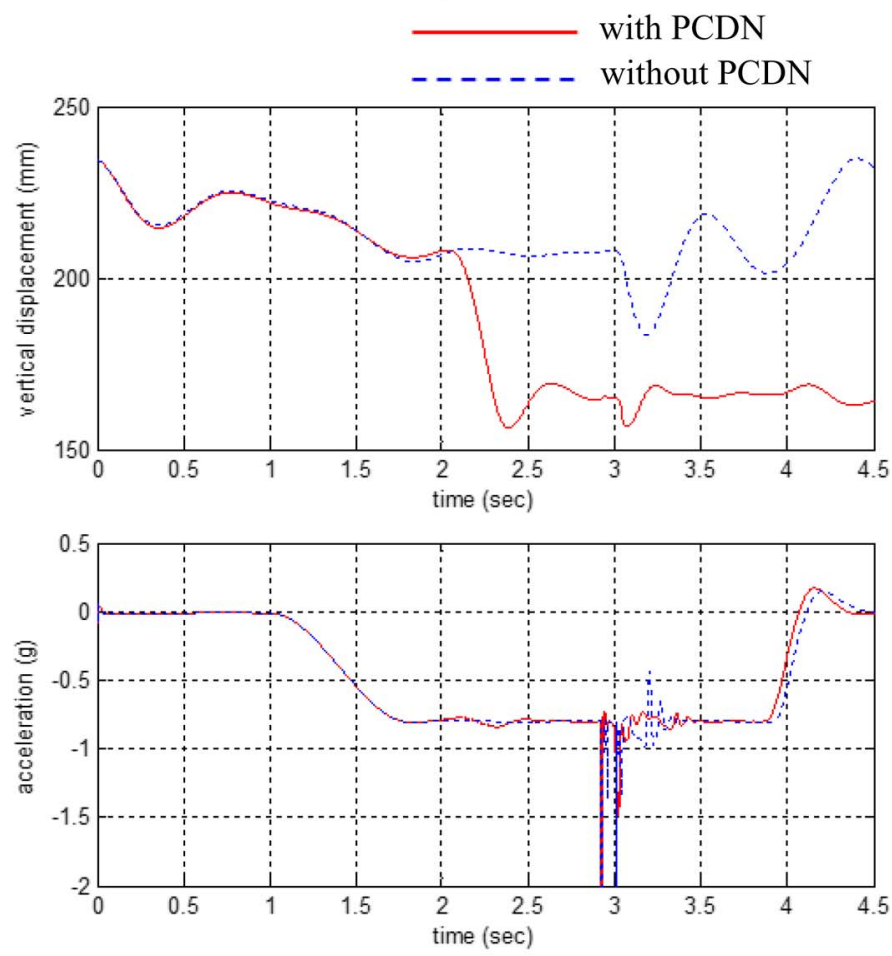

(b)

Fig. 3. Comparison of vertical displacement and acceleration between PCDN and normal case. (a) SUV. (b) Sedan.

passive and forward-dynamic biological models that interact with the environment, tools, equipment, and each other. As it provides Hybrid III crash dummy strength characteristics for passive-type simulations, a reliable human model of an occupant and pedestrian can be constructed for vehicular injury simulation [21]. In our simulations, a Hybrid III crash dummy 

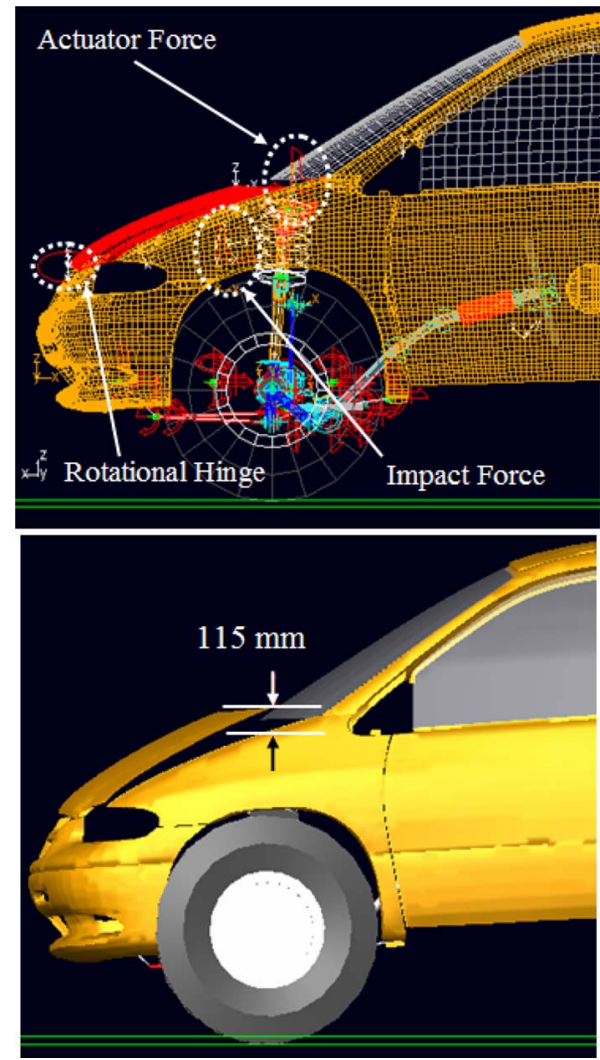

Fig. 4. AHS structure and operation.

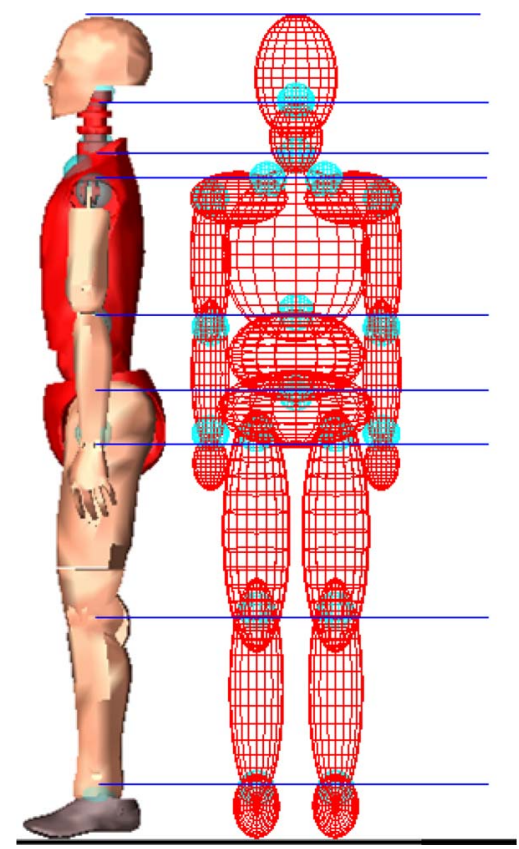

Fig. 5. Pedestrian model based on Hybrid III crash dummy.

with 19 body elements and 18 kinematical joints was used, as shown in Fig. 5.

The body dimensional and inertial property data were produced by the generator of body data (GEBOD) program. These data have applicability to the determination of body mass distribution and human body dynamics modeling and can specif- ically be formatted for use in the articulated total body (ATB) model, which is a 3-D rigid body dynamics simulation program. The data sets generated by GEBOD include the body segments' geometric and mass properties, and the joints' locations and mechanical properties [22]. In our simulations, the subject type was set to a Hybrid III dummy, and the height and weight was set to $170.2 \mathrm{~cm}$ and $68.2 \mathrm{~kg}$, respectively. The generated data were incorporated into the LifeMOD Hybrid III crash dummy model.

\section{Pedestrian InJury CRiterion}

Injury criteria are used to relate quantifiable parameters like acceleration and force to injuries mainly through experiments performed with cadavers. The HIC is based on the premise that the acceleration magnitude coupled with acceleration duration is well correlated with the injury. It is calculated from the resultant head acceleration (measured in $g$ ) like (4), where time is measured in seconds, and the maximum time interval is $36 \mathrm{~ms}$. In (4), $a_{\text {head }}$ denotes the resultant head acceleration in $g$ unit, and $t_{1}$ and $t_{2}$ denote the impact starting and ending times, respectively [23]. The HIC has many shortcomings, but an HIC of 1000 has been associated with a 50\% risk of skull fracture [24]. It was found that the relative frequency of vault and skull base fractures was related with injury severity [25]

$$
\mathrm{HIC}=\max \left(t_{2}-t_{1}\right)\left[\frac{\int_{1}^{2} a_{\text {head }} d t}{\left(t_{1}-t_{2}\right)}\right] .
$$

Accident analyses also proved the importance of head injury [25], [26]. Head, as well as upper and lower extremities, are the most frequently injured body regions in passenger car-topedestrian accidents. However, $62 \%$ of the pedestrian fatalities were caused by head injuries. Brain contusion and skull fractures in general are mainly caused by pedestrian head contact with windscreen/A-pillar and hood [26]. Pedestrian heads suffer both a direct impact with stiff vehicle structures and a large degree of rotational accelerations [27]. Our simulations also used HIC for the measurement of crash severity.

\section{Vehicle-Pedestrian Crash Model}

Considering the statistics of vehicle-pedestrian collisions, a detailed situation was modeled as a crash scenario. The initial speed was set to a relatively high value for an urban roadway, i.e., $75 \mathrm{~km} / \mathrm{h}$, and the initial distance between vehicle and pedestrian was set to $50 \mathrm{~m}$. When the vehicle approaches the pedestrian up to $30 \mathrm{~m}$ in the oncoming direction, the forward obstacle recognition system detects the pedestrian. After a short delay, emergency braking of the vehicle is activated from $25 \mathrm{~m}$ in the front, which is accordant with the general range of collision avoidance/mitigation by braking, i.e., $20-25 \mathrm{~m}$ [27]. Once the emergency braking is activated, the braking force is controlled by the active braking controller to maintain a constant acceleration of $0.8 \mathrm{~g}$. During the time that the vehicle approaches the pedestrian up to $10 \mathrm{~m}$ in front, the forward 

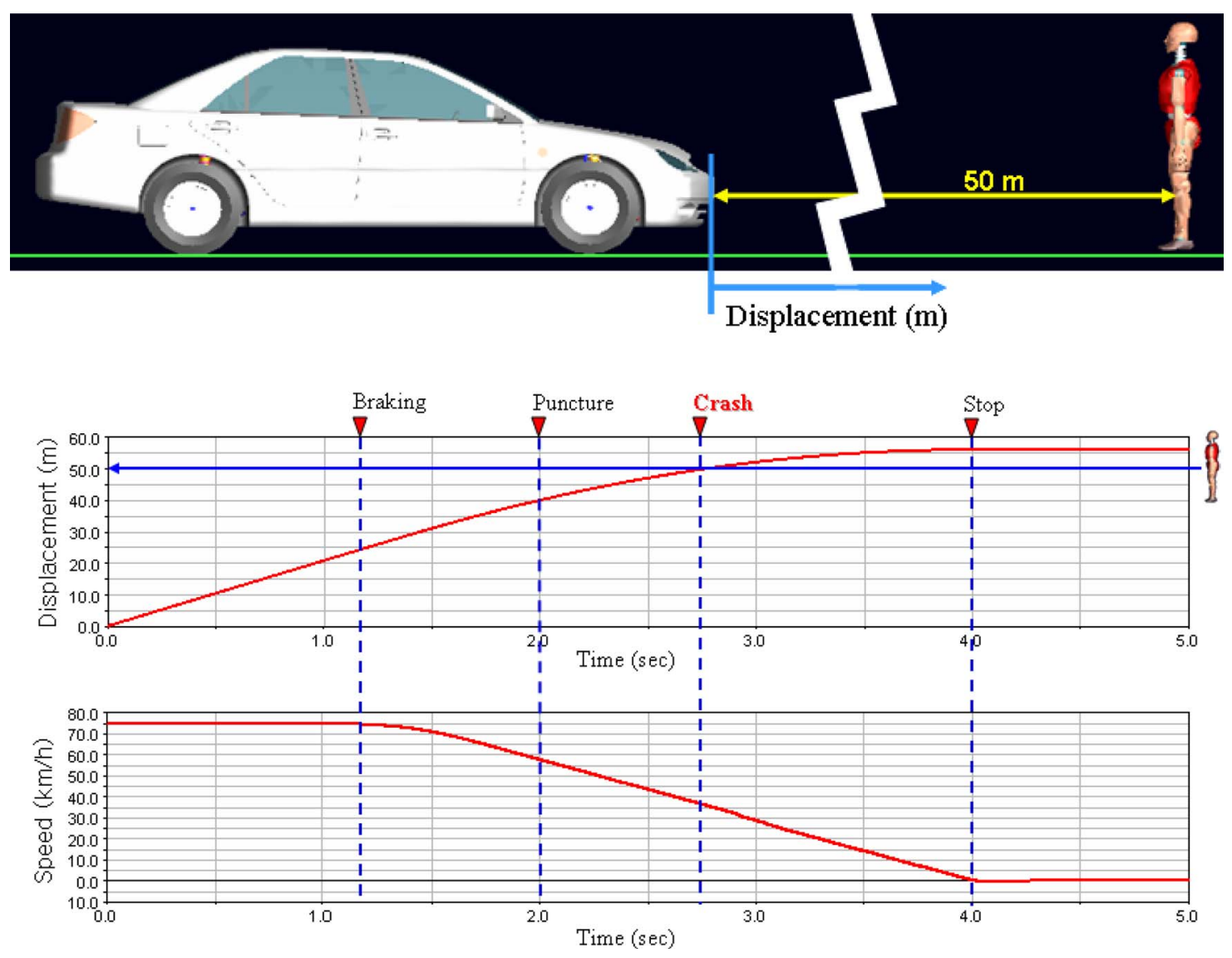

Fig. 6. Vehicle-pedestrian crash scenario.

obstacle recognition system will try to classify the obstacle. When the vehicle reaches the PCDN starting deadline, i.e., $10 \mathrm{~m}$ in front, the forward obstacle recognition system determines the class of the obstacle. In our simulations, we assumed that the forward obstacle recognition system falsely recognized the pedestrian as a side-faced vehicle, and PCDN puncture was activated. It is specifically this type of situation that is of interest in this paper. After $50 \mathrm{~m}$ from the initial position, the vehicle collides with the pedestrian. The collision speed is about $32 \mathrm{~km} / \mathrm{h}$, which is accordant with the fact that $82 \%$ of the accidents happen at an impact velocity of up to $40 \mathrm{~km} / \mathrm{h}$ [27]. This scenario is summarized in Fig. 6.

The contact between objects was modeled by a Hertzian contact stress, which refers to the localized stresses that develop as two curved surfaces come in contact and deform slightly under the imposed loads [28]. According to [29], a pedestrian impact orientation can be classified into five distinctive groups. As the impact speed is about $30 \mathrm{~km} / \mathrm{h}$, and the pedestrian is impacted by the frontal center of the vehicle, the simulation scenario is supposed to be the wrap trajectory [29]. In the wrap trajectory, the pedestrian is struck in the lower legs by the front of a decelerating vehicle. The striking portion of the vehicle must be lower than the height of the pedestrian. Upon impact, the legs buckle, and the torso bends over the hood, and the chest impacts the top of the hood. The head impacts the hood in a whipping motion. After the initial impact, the pedestrian tends to stay on the hood of the car and rides to a stop, sometimes sliding off the hood when the car stops [30].

\section{Simulation Results}

With the constructed models, six cases were simulated and analyzed. Two vehicle models were used (that is, sedan and SUV), and three crash situations were considered: 1) frontal crash with conventional hood (i.e., without AHS); 2) frontal crash with AHS; and 3) side crash without AHS.

\section{A. Sedan-Pedestrian Collision Results}

1) Sedan/Frontal/Without AHS: Fig. 7(a) and (b) shows the crash situations when the PCDN puncture operation is not activated and activated, respectively. In the figures showing the lower leg impact, it can be observed that the level of impact position is lowered from 460 to $375 \mathrm{~mm}$ by $85 \mathrm{~mm}$. In the figures showing head impact, it can be observed that the head impact position approaches the stiff windshield edge from 141 to $81 \mathrm{~mm}$. Fig. 7(c) reveals the head acceleration. The solid line is for the case when the PCDN puncture is on, and the dotted line is for the case when the PCDN puncture is off. The right plot of the figure magnifies the graph near the crash. When the PCDN puncture is activated, it can be observed that the head acceleration increases. The measured HIC value increases from 2419 to 3070.

2) Sedan/Frontal/With AHS: Fig. 8(a) and (b) shows the crash situations when the PCDN puncture operation is not activated and activated, respectively. In the figures showing the lower leg impact, it can be observed that the level of impact 

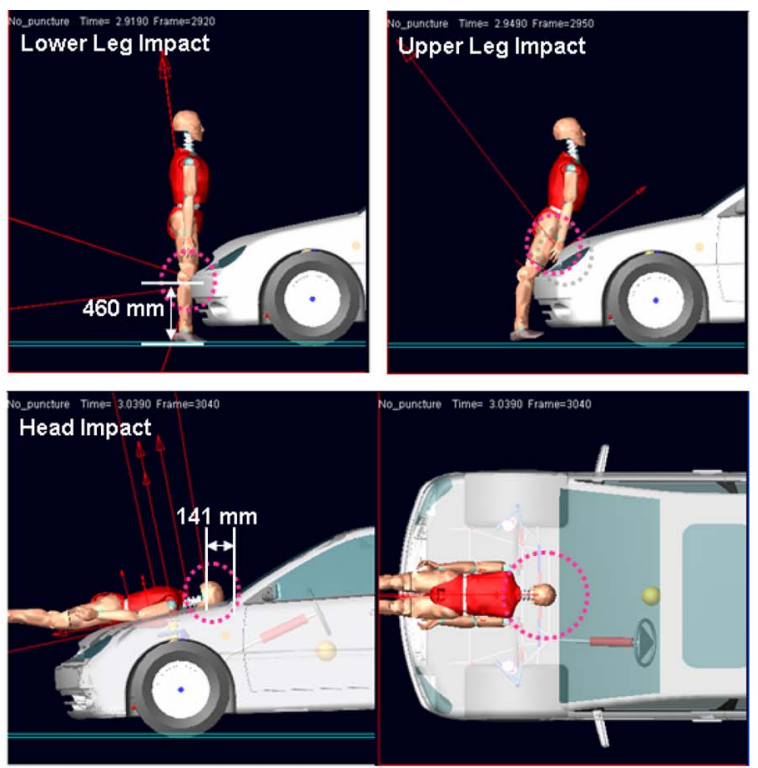

(a)
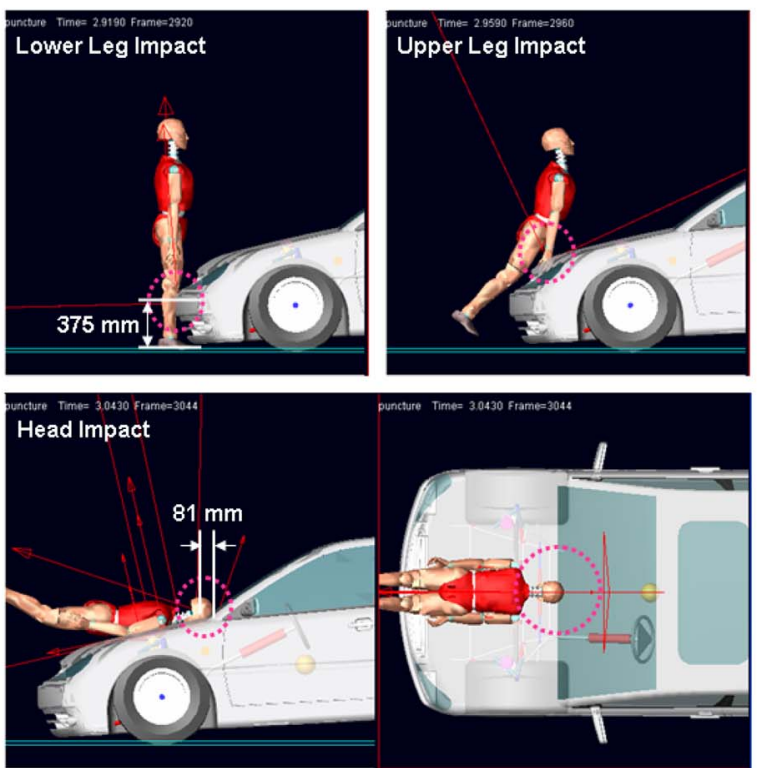

(b)

PCDN puncture on

------ PCDN puncture off

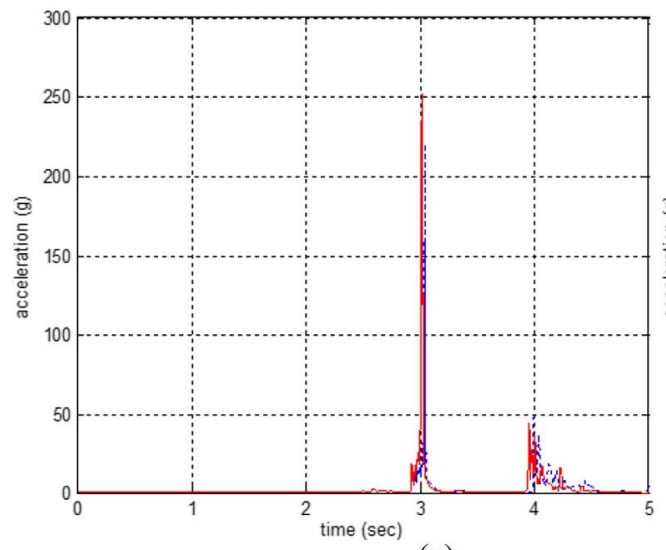

(c)

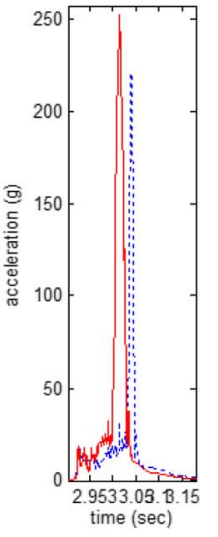

time (sec)
Fig. 7. Simulation result of sedan-pedestrian collision without AHS. (a) PCDN puncture off. (b) PCDN puncture on. (c) Head acceleration comparison.
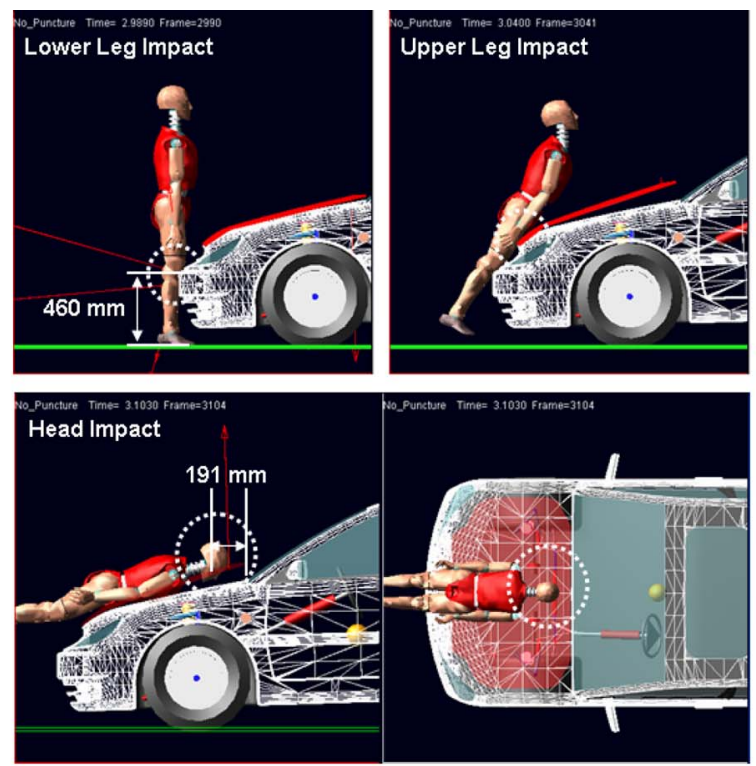

(a)
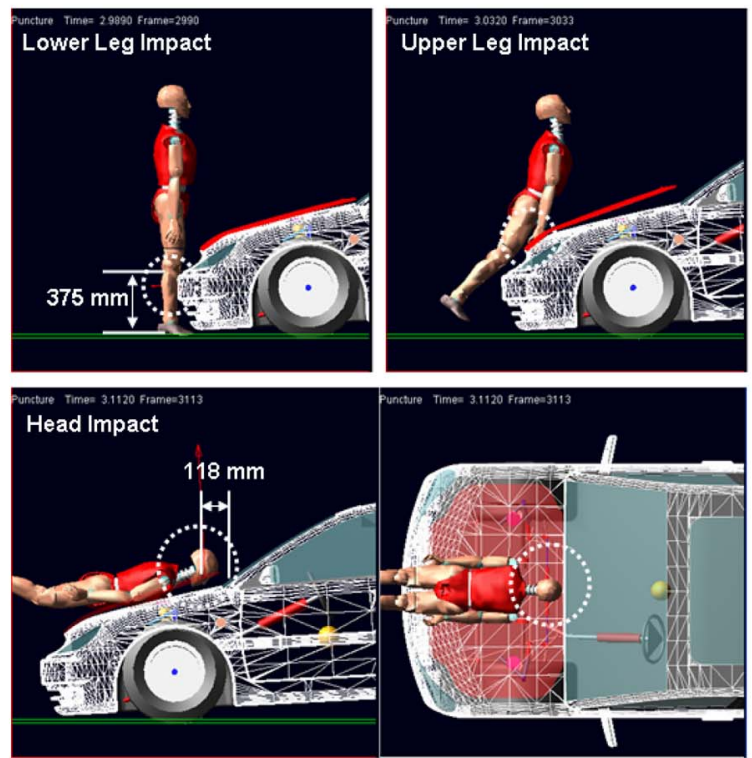

(b)

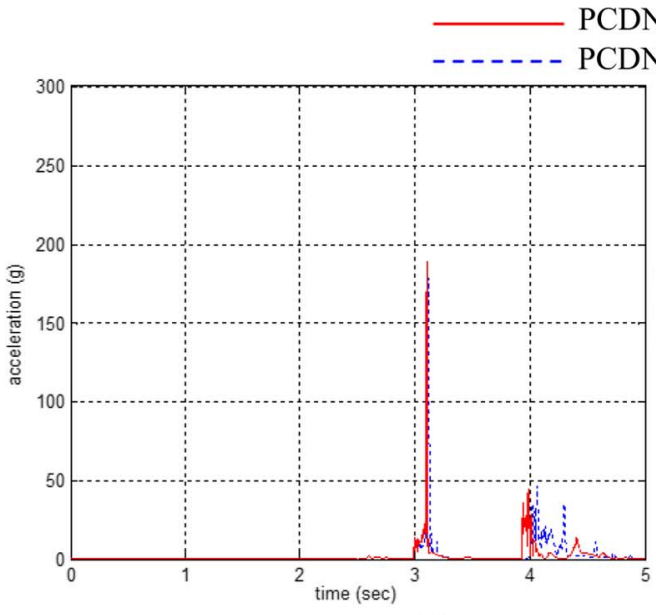

(c)

Fig. 8. Simulation result of sedan-pedestrian collision without AHS (a) PCDN puncture off. (b) PCDN puncture on. (c) Head acceleration comparison. 
position is lowered from 460 to $375 \mathrm{~mm}$ by $85 \mathrm{~mm}$. In the figures showing head impact, it can be observed that the head impact position approaches the stiff windshield edge from 191 to $119 \mathrm{~mm}$. Fig. 8(c) reveals the head acceleration. The solid line is for the case when the PCDN puncture is on, and the dotted line is for the case when the PCDN puncture is off. The right plot of the figure magnifies the graph near the crash. When the PCDN puncture is activated, it can be observed that the head acceleration increases. The measured HIC value increases from 753 to 895 . However, it is noteworthy that the simulation result shows the effectiveness of AHS. The HIC values for both cases are maintained to less than 1000 (corresponding to a $50 \%$ risk of skull fracture), and the increased amount of HIC value is also reduced from 651 to 142 .

3) Sedan/Side/Without AHS: Fig. 9(a) and (b) shows the crash situations when the PCDN puncture operation is not activated and activated, respectively. In the figures of the lower leg impact, it can be observed that the level of impact position is lowered from 459 to $375 \mathrm{~mm}$ by $84 \mathrm{~mm}$. In the figures showing head impact, it can be observed that the head impact position approaches the stiff windshield edge from 298 to $92 \mathrm{~mm}$. Fig. 9(c) shows the head acceleration. The solid line is for the case when PCDN puncture is on, and the dotted line is for the case when PCDN puncture is off. The right plot of the figure magnifies the graph near the crash. When PCDN puncture is activated, it can be observed that the head acceleration increases. The measured HIC value increases from 948 to 1636 .

4) Summary and Analysis: Table I summarizes the simulation results conducted with the sedan and compares the measured HIC values to investigate the effect of PCDN. In all three crash types, when the PCDN puncture operation was activated, the head acceleration and HIC value substantially increased. Particularly, in the side crash situation, the increased amount of HIC value was significantly large.

Such phenomena are thought to be caused by two factors: the height of low leg impact position and the distance between head impact position and windshield edge. In all three crash types, when the PCDN puncture operation was activated, the low leg impact position became lower. Kinematically, this signifies the increase of distance between impact position and the body's center of gravity. Consequently, even with the same impact force, a larger rotational acceleration was caused. Furthermore, in all three crash types, the head impact position approached the windshield edge, which is indicated as the major threat in a pedestrian-vehicle crash because of its stiffness [25].

\section{B. SUV-Pedestrian Crash Simulation Model}

Table II summarizes the simulation results conducted with the SUV and compares the measured HIC values to investigate the effect of PCDN. Like the simulation results using the sedan, in all three crash types, the head acceleration and HIC value substantially increased when the PCDN puncture operation was activated. Particularly, in a side crash situation, the increased HIC value was significantly large.

Because these results are thought to happen for the same reasons as the situations of the sedan, detailed simulation results
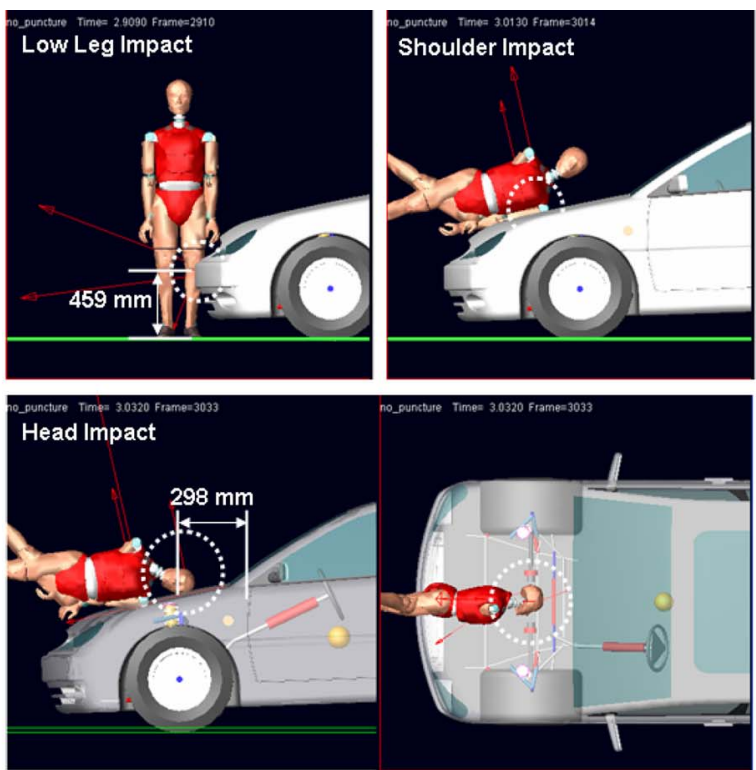

(a)
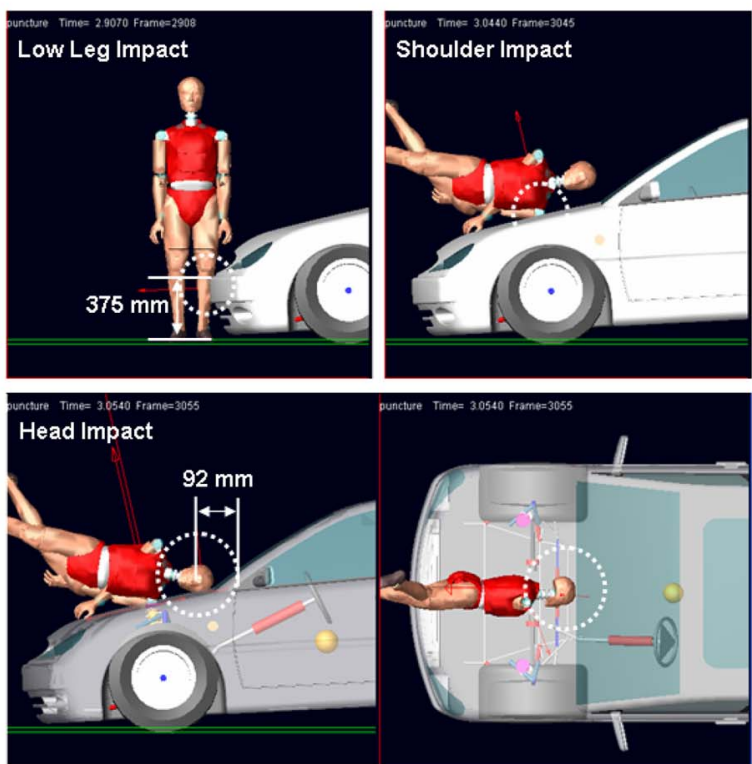

(b)

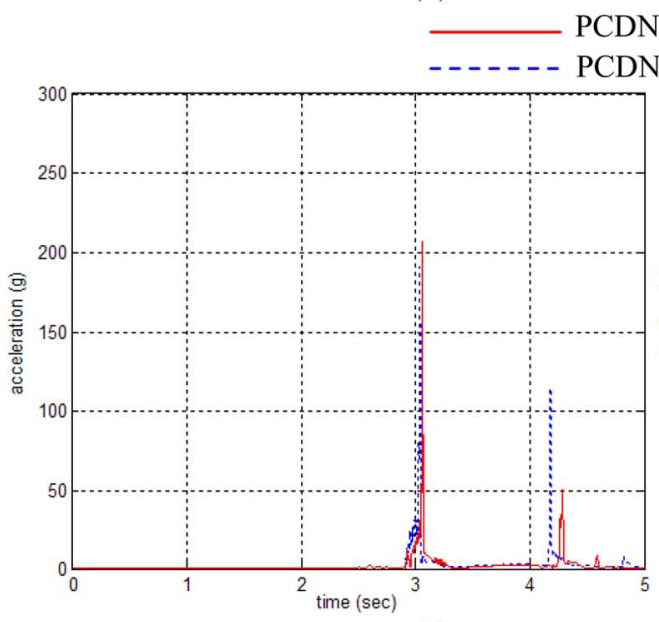

(c)

Fig. 9. Simulation result of sedan-pedestrian collision without AHS. (a) PCDN puncture off. (b) PCDN puncture on. (c) Head acceleration comparison. 
TABLE I

Summary of SEDAN-PEdEstrian CRASh Simulations

\begin{tabular}{|c|c|c|c|c|c|}
\hline \multirow[b]{2}{*}{ Crash type } & \multicolumn{2}{|c|}{ PCDN Puncture Off } & \multicolumn{2}{|c|}{ PCDN Puncture On } & $B-A$ \\
\hline & $\begin{array}{c}\text { Head Acc. } \\
\text { Max }(g)\end{array}$ & $\begin{array}{c}\mathrm{HIC} \\
A\end{array}$ & $\begin{array}{l}\text { Head Acc. } \\
\text { Max }(g)\end{array}$ & $\begin{array}{c}\mathrm{HIC} \\
B\end{array}$ & $\begin{array}{c}A \\
(\%)\end{array}$ \\
\hline Frontal/without AHS & 220 & 2419 & 252 & 3070 & $26.9 \%$ \\
\hline Frontal/with AHS & 177 & 753 & 187 & 895 & $18.8 \%$ \\
\hline Side/without AHS & 188 & 948 & 202 & 1636 & $72.6 \%$ \\
\hline
\end{tabular}

TABLE II

Summary OF SUV-PEDESTRIAN CRASH Simulations

\begin{tabular}{|c|r|r|r|r|r|}
\hline \multirow{2}{*}{ Measures } & \multicolumn{2}{|c|}{ PCDN Puncture Off } & \multicolumn{2}{c|}{ PCDN Puncture On } & \multicolumn{1}{c|}{$\begin{array}{c}\text { PC } \\
\text { Crash type }\end{array}$} \\
\cline { 2 - 5 } & $\begin{array}{c}\text { Head Acc. } \\
\text { Max }(g)\end{array}$ & $\begin{array}{c}\text { HIC } \\
A\end{array}$ & $\begin{array}{c}\text { Head Acc. } \\
\text { Max }(g)\end{array}$ & $\begin{array}{c}\text { HIC } \\
B\end{array}$ & $\begin{array}{c}A \\
(\%)\end{array}$ \\
\hline Frontal/without AHS & 179.4 & 1531 & 203.1 & 2477 & $38.2 \%$ \\
\hline Frontal/with AHS & 130.2 & 814 & 169.3 & 1161 & $43 \%$ \\
\hline Side/without AHS & 230.8 & 1642 & 292.6 & 3979 & $142.3 \%$ \\
\hline
\end{tabular}

are omitted. In all three crash types, the low leg impact position became lower, and the head impact position approached the windshield edge closer when the PCDN puncture operation was activated. However, it is noteworthy that, as in the simulations with the sedan, AHS showed its feasibility by significantly reducing the HIC compared with normal hood structure.

\section{Discussion}

Considering the simulation results, collectively, we can conclude that the PCDN puncture operation makes the struck pedestrian's injury worse. Particularly, in side crash situations, the PCDN puncture operation significantly increases the HIC. Considering the fact that $90 \%$ of the pedestrians were struck from lateral directions [25], the negative effect of PCDN in a side crash situation is fatal.

Shin et al.'s results [31] are coincident with our simulation results in some aspects. They performed multiple simulations and experiments by vertically moving the pedestrian dummy with respect to the vehicle reference frame to evaluate the influence of shifting body contact points with respect to vehicle geometry on impact kinematics. The pedestrian dummy slid farther up the vehicle hood in the upward-shifted model than in the baseline or downward-shifted models. Then, they performed multiple simulations where the center of gravity of the pedestrian was shifted around the baseline to evaluate the contribution of mass distribution with respect to vehicle geometry. As the center of gravity was shifted upward, the head impact position moved farther from the front end of the vehicle and approached the windshield edge. As the PCDN puncture is supposed to have the same effect as the upwardshifted pedestrian dummy, the movement of the head impact position to the windshield edge appears to be the common result.
However, Simms et al.'s suggestion [23] is the exact opposite of our conclusion. They started from the empirical evidence of SUV risk: Lefler et al.'s research showing that $11.5 \%$ of the pedestrians struck by large SUVs are killed compared with $4.5 \%$ for pedestrians struck by cars based on the real-world data from the United States [32] and Roudsari et al.'s research showing that light truck-type vehicles presented a threefold higher risk of severe injuries to pedestrians than cars [33]. They conducted vehicle-Polar-II dummy crash experiments and simulations to compare the pedestrian injuries with respect to the vehicle types. They concluded that the head injuries were similar or slightly lower from contact with SUVs compared to cars, but injuries to the mid-body regions were substantially higher. They analyzed that the primary reason for the increased hazard to pedestrians from SUVs is the high front shape of the bumper and bonnet, and the mass difference between cars and SUVs is not very significant for pedestrian injury causation [23]. The point where their results meet ours is their prediction or suggestion that lowering the bumper and bonnet and reducing the bonnet stiffness for SUVs would help reduce injuries to these mid-body regions. Consequently, from the perspective of PCDN, they insisted that the PCDN puncture operation will reduce the pedestrian injury in the case of SUVs.

However, we conclude that Simms et al.'s suggestion is incorrect based on two facts. First, although our simulation used only head acceleration and they considered four injuries, there was one common observation that the injury caused by the SUV was higher than by sedan in the case of side collisions without AHS. These can be observed at Tables I and II. In other words, our simulation results already include their results if ignoring the magnitude. Second, the fact that an SUV with stronger material, a larger frontal structure, and a higher frontal level is more dangerous than a sedan does not guarantee that the pedestrian will be in less danger if the SUV lowers its 
frontal level. Such a hasty inference seems to ignore too many differences, except for vehicle height.

\section{CONCLUSION}

This paper has investigated how the PCDN puncture operation influences the struck pedestrian as the crash sensor of PCDN falsely recognizes the pedestrian as a side-faced vehicle. A full vehicle, air spring with PCDN actuator, AHS, pedestrian, and crash situation were modeled. Then, three crash situations were simulated each for a sedan and an SUV: frontal crash without AHS, frontal crash with AHS, and side crash without AHS. Three facts were found. 1) In all situations, the pedestrian injury is substantially worse because of the PCDN puncture operation. 2) Particularly, pedestrian injury by side crash is significantly worse than in a frontal crash. 3) AHS substantially reduces not only pedestrian injury but also the increased severity of the injury by PCDN puncture operation.

There is no sensing system without detection/recognition error. Therefore, it is very important to investigate the situation when the sensing system makes a mistake. As shown in the airbag tragedies involving children and short drivers, consideration of unintended situations is essential and important for safety-related systems. Pedestrians seem to be an unintended but important threat to PCDN. In this sense and based on the simulation results, we insist that PCDN should incorporate a pedestrian recognition capability into its sensing system to exclude pedestrians from its interesting obstacles. Additionally, considering the fact that AHS significantly reduced pedestrian injury and the severity of injury caused by PCDN puncture operation, we suggest a complementary method that activates AHS when PCDN is activated.

Although a system is developed only for vehicle-vehicle crash, it could be a threat to pedestrian safety. To be more precise, it should be investigated in the perspective of pedestrian safety and should be proved to be not harmful to pedestrians.

\section{REFERENCES}

[1] E. Zimmerman, "Pre-crash actuator to improve car structural performance in side impact," in Proc. 11th Eur. Automotive Congr., May 30Jun. 1, 2007. [CD-ROM].

[2] IIHS, "Side airbags that protect the head reduce driver fatality risk by 45 percent," IIHS Status Rep., vol. 38, no. 8, pp. 1-3, Aug. 26, 2003.

[3] H. Huh, J. H. Lim, J. H. Song, K.-S. Lee, Y.-W. Lee, and S. S. Han, "Crashworthiness assessment of side impact of an auto-body with 60TRIP steel for side members," Int. J. Automot. Technol., vol. 4, no. 3, pp. 149156, 2003.

[4] F. Zeidler, "The passive safety of passenger cars-Status 2007," in Proc. 11th Eur. Automotive Congr., May 30-Jun. 1, 2007. [CD-ROM].

[5] IIHS, "Side airbags are reducing driver deaths in both cars and SUVs," IIHS Status Rep. vol. 41, no. 8, pp. 1-3, and 7, Oct. 7, 2006.

[6] M.-M. Meinecke, R. Holze, M. Gonter, T. Wohllebe, R. Mende, and R. Petelka, "Side-pre-crash sending system for automatic vehicle height level adaptation," in Proc. 3rd Int. Workshop Intell. Transp., Hamburg, Germany, Mar. 14-15, 2006.

[7] S. Arduc, R. Justen, and T. Unselt, "Motor vehicle with a pre-safesystem," U.S. Patent US2005/0080530 A1, Apr. 14, 2005.

[8] Autoliv, Exciting Project Portfolio, Pre-Crash Dipping Nose. [Online]. Available: http://autoliv.hhit.com/annualreport2003/about/ exciting_project_portfolio.asp

[9] B. De Mersseman, S. D. Barbat, C. J. Sherwin, and S. W. Decker, "Precrash nose dipping system," U.S. Patent US 6993422 B2, Jun. 31, 2006.
[10] F. Kruse, F. Fölster, and M. Ahrholdt, "Target classification based on neardistance radar sensors," in Proc. IEEE Intell. Vehicle Symp., Jun. 14-17, 2004, pp. 722-727.

[11] M. K. Rao, N. V. Smirnov, K. O. Prakah-Asante, and G. S. Strumolo, "Method for determining a danger zone for a pre-crash sensing system in a vehicle having a countermeasure system," U.S. Patent US 6728617 B2, Apr. 27, 2004.

[12] M. E. Farmer and A. K. Jain, "Smart automotive airbags: Occupant classification and tracking," IEEE Trans. Veh. Technol., vol. 56, no. 1, pp. 60-80, Jan. 2007.

[13] T. Gandhi and M. M. Trivedi, "Pedestrian protection system: Issue, survey, and challenge," IEEE Trans. Intell. Transp. Syst., vol. 8, no. 3, pp. 413-430, Sep. 2007.

[14] E. Moxey, N. Johnson, M. G. McCarthy, L. Galloway, G. A. Parker, and W. M. McLundie, "Advanced protection for vulnerable road users: A case study," Proc. Inst. Mech. Eng. Part D: J. Automobile Eng., vol. 220, no. 6, pp. 723-734, 2006.

[15] MSC.Software, Adams; Engineering Productivity Through Advanced Multi-Body Simulation. Accessed at Jan. 15, 2007. [Online]. Available: http://www.mscsoftware.com/assets/MSC_DS_Adams.pdf

[16] J. R. Crandall, K. S. Bhalla, and N. J. Madeley, "Designing road vehicles for pedestrian protection," Br. Med. J., vol. 324, no. 7346, pp. 1145-1148, May 11, 2002.

[17] C. Kerkeling and J. Schäfer, "Structural hood and hinge concepts for pedestrian protection," in Proc. 19th Int. Tech. Conf. Enhanced Safety, Washington, DC, Jun. 6-9, 2005, pp. 379-389.

[18] R. Fredricksson and Y. Håland, Evaluation of a New Pedestrian Head Injury Protection System With a Sensor in the Bumper and Lifting of the Bonnet's Rear Part. [Online]. Available: http://www.autoliv.com/ alv/resources/file/eb3a4d4371424ab/Pedestrian.pdf

[19] M.-K. Shin, K.-T. Park, K.-B. Lee, H.-I. Bae, and G.-J. Park, "Design of the active hood lift system using orthogonal arrays," Trans. KSAE, vol. 14, no. 4, pp. 123-131, 2006 (in Korean).

[20] K. Lee, H.-I. Bae, and H.-T. Kim, "The study on developing active hood lift mechanism for decreasing pedestrian head injury," in Proc. KSAE Spring Conf., 2005, pp. 706-710 (in Korean).

[21] Biomechanics Research Group, Inc., Exploring the Performance of the Biomechanical Machine With Physics-Based Simulation. Accessed on Jan. 17, 2007. [Online]. Available: http://www.lifemodeler.com

[22] H. Cheng, L. Obergefell, and A. Rizer, "The development of the GEBOD program," in Proc. 15th Southern Biomed. Eng. Conf., Mar. 19-31, 1996, pp. $251-254$.

[23] C. K. Simms and D. P. Wood, "Pedestrian risk from cars and sport utility vehicles-A comparative analytical study," Proc. Inst. Mech. Eng. Part D: J. Automobile Eng., vol. 220, no. 8, pp. 1085-1100, 2006.

[24] E. Hertz, "A note on the head injury criterion as a prediction of skull fracture," in Proc. 37th AAAM Conf., San Antonio, TX, 1993.

[25] J. Yao, J. Yang, and D. Otte, "Investigation of head injuries by reconstructions of real-world vehicle-versus-adult-pedestrian accidents," Saf. Sci., vol. 46, no. 7, pp. 1103-1114, Aug. 2008. DOI:10.1016/ j.ssci.2007.06.021.

[26] W. Koch and M. Howard, "Comprehensive approach to increased pedestrian safety in pedestrian-Car accidents," in Proc. Inst. Mech. Eng. Part D: J. Automobile Eng., Jul. 2003, vol. 217, pp. 513-519.

[27] R. Fröming, V. Schindler, and M. Kühn, "Requirement engineering for active safety pedestrian protection systems based on accident research," in Advanced Microsystems for Automotive Applications 2006. New York: Springer-Verlag, Apr. 25-26, 2006, pp. 79-106.

[28] J. E. Shigley and C. R. Mischke, Mechanical Engineering Design, 5th ed. New York: McGraw-Hill, 1989.

[29] J. J. Eubanks, Pedestrian Accident Reconstruction. Tucson, AZ: Lawyers \& Judges, 1994.

[30] L. Martinez, Pedestrian Accident Reconstruction-Review and Update. Accessed at Jan. 18, 2008. [Online]. Available: http://www.tarorigin. com/art/Lmartinez/Ped/

[31] J. Shin, C. Untaroiu, J. Kerrigan, J. Crandall, D. Subit, Y. Takahashi, A. Akiyama, Y. Kikuchi, and D. Longhitano, "Investigating pedestrian kinematics with the polar-II finite element model," presented at the SAE Congr., Detroit, MI, 2007, SAE Paper 2007-01-0756.

[32] D. E. Lefler and H. C. Gabler, "The fatality and injury risk of light truck impacts with pedestrians in the United States," Accident Anal. Prev., vol. 36, no. 2, pp. 295-304, Mar. 2004.

[33] B. S. Roudsari, C. N. Mock, R. Kaufman, D. Grossman, B. Y. Henary, and J. Crandall, "Pedestrian crashes: Higher injury severity and mortality rate for light truck vehicles compared with passenger vehicles," Inj. Prev., vol. 10, no. 3, pp. 154-158, Jun. 2004. 


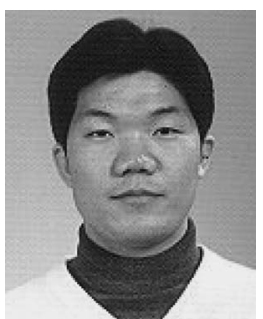

Ho Gi Jung (M'05) received the B.S., M.S., and $\mathrm{Ph} . \mathrm{D}$. degrees in electronic engineering from Yonsei University, Seoul, Korea, in 1995, 1997, and 2008, respectively.

Since 1997, he has been with MANDO Corporation Global R\&D H.Q., Yongin, Korea. He is also with Yonsei University. From 1997 to 2000, he developed environment-recognition algorithms for a lane departure warning system (LDWS) and adaptive cruise control (ACC). From 2000 to 2004, he developed an electronic control unit (ECU) and embedded software for an electrohydraulic braking (EHB) system. Since 2004, he has developed environment-recognition algorithms for an intelligent parking assist system (IPAS), collision warning and avoidance, and an active pedestrian protection system (APPS). His interests are automotive vision, embedded software development, a driver assistant system (DAS), and an active safety vehicle (ASV).

Mr. Jung is a member of the International Society of Automotive Engineering (SAE) and SPIE, which is an international society advancing an interdisciplinary approach to the science and application of light. He is also a member of the Institute of Electronics Engineers of Korea (IEEK) and the Korean Society of Automotive Engineering (KSAE).

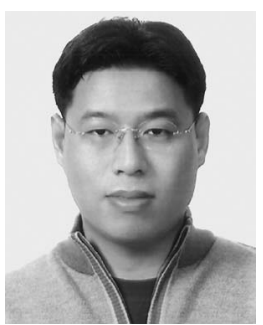

Byung Moon Kwak received the B.S. and M.S. degrees in mechanical engineering from Myongji University, Seoul, Korea, in 1998 and 2000, respectively.

Since 2007, he has been with TUR Consulting Corporation, Suwon, Korea. His interests are dynamic system modeling, control systems, and a hardware-in-the-loop system (HILS).

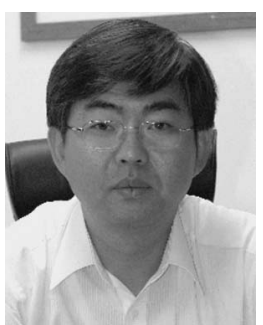

Jeong Soo Shim received the B.S. degree in mechanical engineering from Hanyang University, Seoul, Korea, in 1983 and the M.S. and Ph.D. degrees in mechanical engineering from the Korea Advanced Institute of Science and Technology (KAIST), Deajeon, Korea, in 1985 and 1993, respectively.

Since 2007, he has been a Representative Director with TUR Consulting Corporation, Suwon, Korea. His interests are dynamic system modeling, control systems, mechanical system vibration modeling/ analysis, and mechanism design.

Mr. Shim is a member of the Korean Society of Automotive Engineering (KSAE).

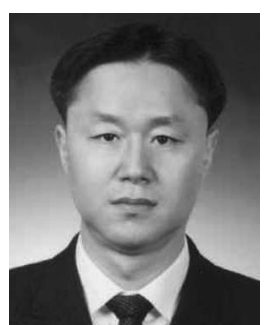

Pal Joo Yoon received the B.S., M.S., and Ph.D. degrees in mechanical engineering from Hanyang University, Seoul, Korea, in 1987, 1989, and 2000, respectively.

Since 1988, he has been with MANDO Corporation Global R\&D H.Q., Yongin, Korea. He has mainly been involved with automotive control system design. He is currently the Head of the System R\&D Center. His interests are a driver assistant system (DAS), autonomous driving, and automotive control system design.

Dr. Yoon is a member of the International Society of Automotive Engineering (SAE) and the Korean Society of Automotive Engineering (KSAE).

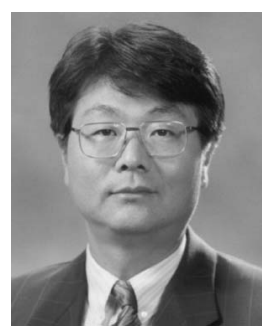

Jaihie Kim received the B.S. degree in electronic engineering from Yonsei University, Seoul, Korea, in 1979 and the M.S. degree in data structures and the $\mathrm{Ph} . \mathrm{D}$. degree in artificial intelligence from Case Western Reserve University, Cleveland, OH, in 1982 and 1984 , respectively.

Since 1984, he has been a Professor with the School of Electrical and Electronic Engineering, Yonsei University, where he is also currently the Director of the Biometric Engineering Research Center (BERC). His research areas include biometrics, computer vision, and pattern recognition.

Prof. Kim is currently the Chairman of the Korean Biometric Association and the President of the Institute of Electronic Engineers of Korea (IEEK). 